\title{
Überlegungen und zehn Thesen zur Bedeutung der Hochschulen im Zuge der digitalen Revolution
}

Dieser Essay geht von der Grundannahme aus, dass die Hochschulen bei der Gestaltung der digitalen Revolution eine tragende Rolle einnehmen können und sollten. Die Verantwortung, die ihnen in diesem Prozess zukommt, ist überragend. Inmitten des paradigmatischen digitalen Wandels kann es allerdings nicht allein darum gehen, die bisherigen Formen und Inhalte von Bildung und Forschung einfach zu digitalisieren. Sie bedürfen vielmehr einer grundsätzlichen Transformation, damit die Hochschulen nicht nur die Zukunft gestalten können, sondern um selbst überhaupt eine Zukunft haben.

Deshalb dürfte es einerseits viel zu kurz gegriffen sein, wenn man ausschließlich aus den bisherigen, größtenteils sehr engen Strukturen heraus versucht, den Anforderungen der Digitalisierung irgendwie gerecht zu werden. Andererseits ist es ebenso wenig damit getan, disruptiv alle Hochschulstrukturen preiszugeben und ultimativ zu zerschlagen. Wenn bei dem digitalen Transformationsprozess Bewahrendes und Innovatives miteinander zu verbinden sind, gleicht das im Anspruch einer Quadratur des Kreises. Diese Anforderung erfordert deshalb die Fähigkeit, sich auf den Weg zu machen und mit der Arbeit zu beginnen, obwohl für viele Teilaufgaben noch lange keine Lösungen in Sicht sind und die Entwicklungsziele noch gar nicht in Gänze vorstellbar sein können. Dies auszuhalten braucht den Mut, immer den ganz großen Wurf, wenn schon nicht im Blick, so doch in der Vorstellung zu haben. Aber sie bedarf auch der Bescheidenheit, den Auswüchsen der Zerstörungswut digitaler Disruptivität zu widerstehen. Letztere führt bisweilen längst dazu, die Existenzberechtigung von Bildungseinrichtungen im Allgemeinen und Hochschulen im Besonderen komplett in Frage zu stellen.

Hier wird von der Prämisse ausgegangen, dass wir Hochschulen mehr denn je brauchen, gerade weil sie die beste Expertise dafür bieten, Tradiertes und Innovatives sinnvoll miteinander in produktiver Dissonanz und Resonanz zu bringen. Dies gelingt ihnen nicht zuletzt gerade deshalb, weil sich Wissenschaft im besten Falle immer wieder selbst in Frage zu stellen vermag. Kein ultimatives Ziel zu kennen, immer im Fluss zu 
sein und sich deshalb auch in Bescheidenheit zu üben, gehört zu ihrem Wesen. Widersprüchlichkeit und Widerständigkeit in der Freiheit von Forschung und Lehre auszuhalten, ist ihre Kernkompetenz. Dazu gehört es auch - heute gerade mehr denn je -, dass nichts so unbeständig ist, wie das gerade vermeintlich gesicherte Wissen von heute.

Allerdings dürfte nichts die Wissenschaft weiter voranbringen als die digitale Revolution, man denke nur an die moderne Genetik und die Neuroinformatik. Jenseits der Forschung scheint aber auch kaum etwas die Hochschullandschaft und ihre Bildungsaufträge so sehr in Frage zu stellen, wie die Digitalisierung. Wenn es also um die ganz großen Würfe aus einer noch ziemlich nebulösen Umgebung geht, dann müssen Thesen her. Dieser Essay beinhaltet Vorschläge für zehn solcher, vielleicht hilfreicher Arbeitshypothesen, dies ohne Anspruch auf eine ultimative Treffsicherheit, geschweige denn auf Vollständigkeit.

Die Hochschulen selbst müssen zu Inkubatoren der digitalen Revolution werden. Für eine so zukunftsorientierte Veranstaltung wie das Hochschulwesen muss die Angst vor dem Neuen geringer ausfallen, als die Angst etwas Neues zu verpassen. Insofern steht es außer Frage, dass es der Hochschule hauptsächlich darum gehen könnte, die Digitalisierung vornehmlich zu bremsen. Sie muss es sich vielmehr selbst zum Ziel machen, die digitale Revolution in die Hand zu nehmen und zu gestalten. Dies kann nicht allein der Wirtschaft überlassen werden. Diese würde es auch ohne die Hochschulen tun, dann aber ohne Regulativ. Denn auch die Politik ist auf Impulse der Wissenschaft angewiesen, um die Digitalisierung in gute Bahnen zu lenken. Wer etwas mitgestalten will, muss allerdings eigene Triebkräfte einbringen. Die Hochschulen als Institutionen und alle ihre Fachdisziplinen sollten den Digitalisierungsimpuls aufnehmen und selbst weitertragen, dies aber nicht aus wirtschaftlichem Interesse, sondern aus dem Durst nach Erkenntnis und der Überzeugung, dass es Mensch und Natur zum Guten gereichen könnte. Dafür bedarf es nicht nur der Natursondern auch der Geisteswissenschaften. 
Die Hochschulen müssen wieder zu wirtschaftlich und politisch unabhängigen Thinktanks werden. In ihren besten Zeiten und in den progressivsten Gesellschafen fungierten Universitäten stets als herausragende Impulsgeber für gesellschaftliche Entwicklungen. Ihre Universalität und ihre Unabhängigkeit versetzen sie in die Lage, in weit offenen Spielräumen und weit vorausdenkenden Horizonten zu denken und zu experimentieren, um die gesellschaftliche Entwicklung schließlich auch auf der Handlungsebene voranzubringen. Dies geht soweit, dass in der Vergangenheit von Universitäten sogar Revolutionen ausgegangen sind, dies freilich sowohl aus ihnen heraus als auch aus ihrer Peripherie.

In den letzten Jahrzehnten scheinen diejenigen Denkfabriken, von denen ein revolutionärer Impact ausgeht, eher im Sektor der Wirtschaft $\mathrm{zu}$ verorten zu sein, ursprünglich vor allem im sogenannten militärischindustriellen Komplex und nun vor allem in der IT-Branche, insbesondere in ihrem Epizentrum, dem Silicon Valley. Die ökonomische Dimension der digitalen Revolution schien zunächst untrennbar verbunden mit dem, was wir heute unter globalem Neoliberalismus verstehen. Es braucht kaum betont zu werden, dass sich demokratische Gesellschaften nicht darauf verlassen können, dass der Markt auch das politische Geschäft gut zu regeln vermag. Vor dem, was Gesellschaften und Staaten zusammenhält, machen die global operierenden IT-Giganten allerdings nicht Halt, wenn sie nach immer fundamentaleren Disruptionen trachten. Längst haben sich beispielsweise an den Hochschulen vorbei Bildungseinrichtungen entwickelt, die das Versprechen in sich tragen, Studiengänge besser, innovativer und effizienter zu gestalten. Und immer mehr Heranwachsende und Unternehmen scheinen ihnen das auch zuzutrauen. Es wird Zeit, dass sich die Universitäten ihre Kernkompetenz als Thinktanks zurückholen und sich daraus ihre Existenzberechtigung sichern. $\mathrm{Ob}$ dies gelingt, dürfte entscheidend davon $\mathrm{ab}-$ hängig sein, in wie weit die Unterschiede und Gemeinsamkeiten zu den Thinktanks der Industrie transparent herausgearbeitet werden können. Dazu ist es wichtig, dass die Universitäten erstens budgetär in die Lage versetzt werden, um kluge Köpfe zu konkurrieren, frei zu forschen, um sich zweitens aber auch mit dem Entdeckten und Entwickelten irgendwann auch ökonomisch unabhängig machen zu können, ohne dass die Universitäten - und das ist die größte Schwierigkeit dabei - am Ende in erster Linie zu Inkubatoren für die heißesten Startups werden, die dann wiederum von den IT-Riesen einverleibt werden. 
Die digitale Transformation der Hochschulen sollten zuvorderst die Themen Erziehung und Bildung in den Fokus nehmen. Schlimm genug, dass sich die Hochschulen so viel an denkerischem Potential abnehmen lassen. Schlimmer aber noch wiegt, dass sie ihr früheres Monopol für höhere Bildungswege an die Digitalwirtschaft zu verlieren drohen, die längst die alten Hochschulen infiltrieren oder eigene neue eröffnen. Wenn man davon ausgeht, dass all das, was die Menschheit seit Jahrhunderten an Wissen und Erfahrung zu Erziehung und Bildung generiert hat, mit der Digitalisierung nicht einfach obsolet wird, darf auch hier von einer Kernkompetenz oder gar von einem Hoheitsgebiet ausgegangen werden. Eine der vornehmlichsten Aufgaben der Hochschulen ist es daher, das Lehren, und damit auch das Bilden und Erziehen, zu lehren. Insofern erscheint es als sinnvoll und notwendig, die Digitalisierung zuvorderst in diesen Bereichen konsequent ins Visier zu nehmen. In diesem Zusammenhang wird besonders für die pädagogischen Fachdisziplinen die Notwendigkeit der Verzahnung von Forschung und Lehre besonders evident. Es geht hier um wichtige Kernfragen, die weit für alle Fächer auf die eine oder andere Art und Weise auch relevant sein dürften: In wie weit oder bis zu welchen Alter ist Pädagogik abhängig von einer möglichst unmittelbaren zwischenmenschlichen Beziehungsdimension, wie beispielsweise auch die Medizin und Psychotherapie? Welche Rolle spielen die Präsenz, der Körper und die Sinne, beim Lernen von Kindern, Jugendlichen und Studenten? Wie unterscheiden sich analoges und digital vermitteltes Lernen im Erreichen von Lernzielen? Können mit Hilfe von Gamification und Serious Games bessere Lerneffekte erzielt werden? Die Bewältigung welcher Entwicklungsaufgaben lässt sich digitalisieren und welche sollten vielleicht trotzdem weiter in der Hand des Menschen verbleiben? Und vor allem was muss der heranwachsende Mensch überhaupt noch lernen, welches Wissen und welche Kompetenz, wenn künstliche Intelligenz und Robotik beim Denken und Handeln immer mehr assistieren und übernehmen?

In letzter Konsequenz dürfte es eine der wichtigsten Aufgaben - vor allem, aber nicht allein - der pädagogischen Hochschulen werden, herauszuarbeiten, was wir an Kulturtechniken und Wissen im Sinne von Sicherungskopien und Backups bestenfalls noch im Menschen anlegen sollten, was also auch dann noch zur Verfügung stehen sollte, wenn das Netz und damit im Zweifelsfall alle digitalen Medien ausfallen oder in die falschen Hände geraten. Um den digitalen Impuls an der entscheidenden 
Stelle mitzunehmen und Multiplikatoreneffekte zu schaffen, letztlich aber auch um überhaupt diejenigen zu erreichen, die schon in der digitalen Revolution groß werden, bedarf es hier rasch einer größtmöglichen Anstrengung. Dabei geht es ganz konkret darum, die private und professionelle pädagogische Orientierungslosigkeit angesichts der digitalen Bildungsoffensiven zu überwinden, aber auch darum, den Hochschulen das Überleben zu ermöglichen, damit sie nicht am Ende von der Ökonomie in feindlicher Übernahme in ihrem Wesen disruptiv vernichtet werden.

Die Verflechtung von Wissenschaft und Wirtschaft muss so weit wie möglich transparent gemacht werden und ihre Entflechtung vorangetrieben werden. Damit die Hochschulen eine Chance haben, als eigenständige Instanzen im Rahmen von demokratischen und liberalen Gesellschaften zu existieren, bedarf es klarer Grenzen zwischen den Systemen. Das heißt natürlich nicht, dass es nicht zu Kooperationen und Kollaborationen zwischen Universitäten und Akteuren der Wirtschaft kommen darf, aber es bedarf klarer Spielregeln, unter welchen Bedingungen diese zustande kommen und umgesetzt werden dürfen. Und es bedarf einer konsequenten Transparenz für die Bürgerinnen und Bürger sowie Kontrollinstanzen, um sicherzustellen, dass die im Auftrag der Gesellschaft agierenden Universitäten auch wirklich in ihrem Sinne operieren.

Der ausufernden Metrisierung und Ökonomisierung von Wissenschaft ist Einhalt zu gebieten. Die Ökonomisierung der Hochschullandschaft hat viele Gesichter. Wirtschaftliche und metrische Bemessungsgrundlagen haben überall Einzug gehalten. Nur was zahlenmäßig messbar und/oder digitalisierbar ist, scheint noch Bestand zu haben. Wenngleich sich eine Renaissance qualitativer Forschungsansätze zumindest erahnen lässt, ist die Quantifizierbarkeit zum entscheidenden Bewertungsmaßstab von Wissenschaft geworden. Forschung, die nicht direkt oder indirekt einem ökonomischen Ziel dient, wird immer fragwürdiger. Und die Bemessungsgrundlage mit mehr als fragwürdigen Impact-Faktoren und ähnlichen Auswüchsen treibt unter der Prämisse einer vermeintlichen $\mathrm{Ob}-$ 
jektivierung bizarre Auswüchse, wobei sich auch hier längst ökonomische Interessen von Wissenschaftsverlagen als Motoren von Fehlentwicklungen andienen. Wie in der Wirtschaft setzt sich längst in der Wissenschaft nicht mehr notwendigerweise das Sinnvolle und Bedeutsame durch, sondern das was Punkte und/oder Geld bringt. Viel zu wenig wird dabei also das Normative bedacht, welches die digitale Metrisierung der Welt mit sich bringt.

Wissenschaft und Lehre gehen mehr denn je unabhängige Wege. So wie die Forschung immer mehr individuellen Interessen der Forschenden und der (potentiellen) Firmengründerinnen und -gründer dient, so sollen Studiengänge heute vor allem auf die Arbeit vorbereiten. Fragen, die den Ernst des Lebens jenseits der individuellen Erwerbstätigkeit und damit auch kollektive Fragen beispielsweise zu Gesellschaft und Politik betreffen, sie werden kaum noch in den Universitäten gestellt.

Die Geisteswissenschaften müssen gestärkt werden. Es bedarf neben den $\mathrm{Na}$ turwissenschaften, zu denen letztlich auch die Informatik gehört, dringend einer neuerlichen Stärkung der Geisteswissenschaften. Viele Gründe können für den Eindruck geltend gemacht werden, dass die Geisteswissenschaften in der Hochschullandschaft um ihre Existenzberechtigung ringen müssen. Die beschriebene Metrisierung und Ökonomisierung der Hochschulen dürften nur einen Teil der Entwicklung erklären. In diesem Zusammenhang kann man auch an den vermeintlichen Niedergang des Sozialismus und den vermeintlichen Sieg des Kapitalismus denken, der nicht nur den Neoliberalismus an die Hochschulen getragen hat, sondern den Universitäten auch etwas an Diskursivität genommen hat. Die Beantwortung der entscheidenden Fragen der Menschheit lässt sich vielleicht nicht mit Zahlen oder Geld erledigen. Dies gilt beispielsweise für Fragen nach dem Wesen des Menschen, nach den Regeln der Zwischenmenschlichkeit im Kleinen und Großen, dies gilt ebenso für viele ethische und ästhetische Fragen, letztlich philosophische Fragen. Jenseits des Zählbaren werden gerade die Geisteswissenschaften dazu in der Lage sein, Fragen zu stellen und Antworten zu suchen, welche Werte und Normen die digitale Revolution überdauern, um nicht zu sagen, überleben werden. 
Die Ethikkommissionen und Datenschutzgremien sollten gestärkt werden, damit sie Vorlagen und Referenzrahmen für Politik und Wirtschaft liefern können. Die Fragen, die die naturwissenschaftliche Forschung mit Hilfe der Digitalen Technologien aufwirft, kann sie nicht selbst beantworten. Ohne die Digitalisierung sind beispielsweise die bahnbrechenden Erkenntnisse von Hirnforschung und Genetik gar nicht vorstellbar. Was allein diese beiden Bereiche an Herausforderungen hinsichtlich Biotechnologie, Robotik und Künstlicher Intelligenz bieten werden, wird die Welt vor gravierende Probleme stellen. Wir sollten längst darüber hinaus sein, als Gesellschaft etwas voranzubringen, nur weil es machbar ist und ökonomisch sinnvoll erscheint. Andere Fragen der Nachhaltigkeit im Hinblick auf das $\mathrm{Zu}-$ sammenleben von Mensch und Natur, sie können lediglich unter Hinzuziehung der Geisteswissenschaften beantwortet werden. Sie müssen dahingehend nicht nur erhalten, sondern auch gefördert und weiterentwickelt werden, insbesondere die Philosophie und ihre Derivate. Ethikkommissionen im Allgemeinen und Datenschutzgremien im Besonderen müssten an den Hochschulen eine herausragende Stellung und eigenständige Formatierung bekommen. Sie müssten zu Instanzen reifen, deren Tätigkeitsfeld weit über die Hochschulforschung und -lehre hinausgeht, Leuchttürme müssten sie sein, Vorbilder aber auch Korrektive, dies insbesondere im Hinblick auf Politik und Wirtschaft. Verantwortung ist neu zu verstehen und umzuverteilen. Letztendlich geht es dabei um die Frage, wer in und nach der digitalen Revolution Verantwortung übernimmt.

Die Hochschulen haben sich besonders mit der Frage zu beschäftigen, was von Erde und Mensch nach industrieller und digitaler Revolution übrigbleiben sollte. Die vornehmliche Aufgabe, Hüterin und Wächterin von ethischen Überlegungen und Entscheidungsprozessen zu sein, würde die Hochschulen auch mit dem Ziel betrauen, sich weiter mit der Frage zu beschäftigen, was den Menschen wirklich ausmacht. Diese Zielsetzung scheint jedoch mit der Digitalisierung einer Umformulierung zu bedürfen. Da es nun der individuelle Mensch selbst ist, der im Zentrum der Disruptionsversuche der Digitalisierung steht, indem er von Künstlicher Intelligenz und Robotik immer mehr ersetzt und überholt wird, geht es nunmehr um die 
Frage, was denn von ihm in Zukunft eigentlich übrigbleibt. Das ist eine Frage, die im Grunde einer Kollaboration von Geistes- und Naturwissenschaft bedarf. Für die Zukunft der Hochschulen ist die Annäherung an eine Beantwortung dieser Frage essentiell, um nicht zusagen lebensnotwendig. Wir müssen die Zukunft des Menschen antizipieren, um die nachwachsenden Generationen gut darauf vorbereiten zu können. Wir müssen letztendlich angemessene Sicherungsmaßnahmen ergreifen, wenn wir feststellen sollten, dass es dem Menschen an und für sich an den Kragen geht, vorausgesetzt wir haben wirklich ein Interesse an seinem Erhalt. Den Hochschulen stünde es gut zu Gesicht, sich in dieser Hinsicht vor allem an den Schnittstellen zwischen Natur- und Geisteswissenschaften $\mathrm{zu}$ engagieren, um empathisch und ethisch zu handeln und daraufhin zu arbeiten, den Planeten und möglichst auch den Menschen in seiner Extistenz zu retten.

Die Hochschulen können Synergien von Bewahrendem und Erneuerndem schaffen. Eine solche Rettung von Mensch und Umwelt scheint längst nicht mehr denkbar ohne den Einsatz von Technologien. Bewahrendes und Erneuerndes gegeneinander auszuspielen, gilt es zu überwinden, indem konservative und innovative Impulse synergetisch und integrativ $\mathrm{zu}$ einem konstruktiven Ziel geführt werden. Das ist nicht notwendigerweise eine Quadratur des Kreises. Wenn sich aber überhaupt jemand mit dieser Materie auskennt, dann sind das diejenigen Wissenschaftler und Wissenschaftlerinnen, die sich mit den vermutlich anspruchsvollsten Naturwissenschaften (Quantenphysik) und Geisteswissenschaften (Philosophie) auskennen. In diesem Sinne und Zuge könnten Hochschulen neuerlich zu Glanz und Relevanz auf höchster Ebene kommen.

Hochschulen können zugunsten des Erhalts und der Weiterentwicklung demokratischer Prozesse und Systeme zu Vermittlern zwischen repräsentativer und direkter Demokratie werden. $\mathrm{Zu}$ dieser neuen Bedeutung kommen die Hochschulen allerdings nur dann, wenn sie sich selbst als revolutionäre Figuren verstehen, die über Diskursivität letztendlich zu Vermittlung und Integration finden. Neben dem Erhalt von Natur und Mensch dürften 
und müssten die Hochschulen Verantwortung übernehmen, wenn es um die Weiterentwicklung von Demokratien geht, vorausgesetzt sie verstehen sich nicht nur als Bestandteil sondern sogar auch als Motor demokratischer Prozesse und Systeme. Die Digitalisierung erfasst gerade disruptiv eben auch die demokratischen Gebilde, insbesondere das Prinzip der Repräsentativität. Die Parteiendemokratie gegenüber der sich gerade vornehmlich auf digitalen Wegen rührenden Zivilgesellschaft und ihren neuen Instanzen und Kräften nicht nur zu schützen, sondern sie daran wachsen $\mathrm{zu}$ lassen, beziehungsweise in ein gleichberechtigtes Zusammenspiel zu bringen, dies könnte auch eine Aufgabe der Hochschulen sein, sowohl indem sie darüber öffentlich nachdenken und debattieren, aber auch indem sie diese Prozesse selbst erproben und mitgestalten. 



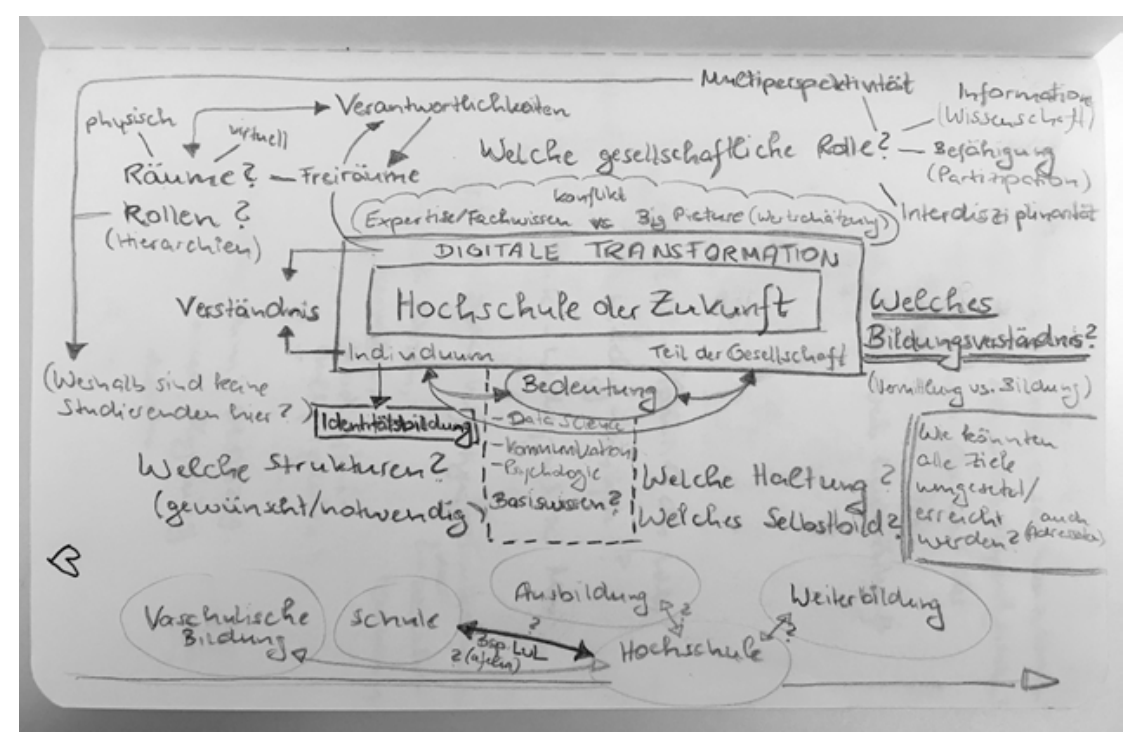


